\title{
Predictors of mortality in patients with severe COVID-19 pneumonia — a retrospective study
}

\begin{abstract}
Introduction: The novel coronavirus pandemic has caused significant mortality throughout the world. This study was done as there is scarce data on mortality predictors in severe COVID-19 pneumonia patients admitted to ICU in the Indian population. Material and methods: A retrospective study was conducted on COVID-19 pneumonia patients admitted to tertiary care center during June-0ctober 2020. The records of patients admitted to ICU were collected and data included demography, symptoms, comorbidites and vital parameters. Laboratory parameters included complete hemogram, random blood sugar, serum ferritin and $\mathrm{LDH}$, renal function test, liver function test. Treatment-associated information such as the use of remdesivir, timing of initiating remdesivir after the symptom onset, the use of steroids, use of anticoagulants, use of HFNC, NIV, ventilator were collected. 30 days mortality data post-discharge was collected via telephonic interview.

Results: 4,012 confirmed cases of COVID-19 were admitted to hospital, of which 560 (13.95\%) with severe pneumonia were included in the study. Mean age was $57.75 \pm 13.96$ years. The mortality rates were $54.64 \%$ among severe COVID- 19 cases and $5 \%$ among mild to moderate COVID-19 cases. The Cox multinominal regression analysis identified $\mathrm{SpO}_{2} / \mathrm{FiO}_{2}<400$, age $>50$ years, duration of symptom $>4$ days, serum ferritin $>450 \mu \mathrm{g} / \mathrm{L}$, respiratory rate $>23 / \mathrm{min}$, the presence of comorbidities and non-usage of remdesivir were independently associated with increased mortality. Mortality rate at 30 days was $56.60 \%$.

Conclusion: Severe COVID-19 pneumonia is associated with very high mortality, especially in a resource-constrained setting. The use of remdesivir may have to be considered early in the course of disease to prevent excess mortality related to COVID-19.
\end{abstract}

Key words: COVID-19, pneumonia, mortality, remdesivir

\section{Introduction}

The novel coronavirus disease (COVID-19) has drew worldwide attention by causing the first pandemic by coronavirus leading to international public health emergency. On the $3^{\text {rd }}$ November, 2020, the World Health Organization (WHO) declared coronavirus outbreak as pandemic and public health emergency of international concern [1]. SARS-CoV-2 infection has caused significant morbidity and mortality throughout the world leading to immense health care burden. Currently, worldwide around 55 million people have been infected with SARS-CoV-2, which has re- sulted in around 1.35 million deaths [2]. In India, around 9 million people have been infected and approximately 132 thousand people have succumbed to SARS-CoV-2 infection [3]. Very little attention has been paid to clinical characteristics and outcomes of severe COVID-19 pneumonia patients in intensive care unit (ICU), data on whom are scarce but are of paramount importance to reduce mortality in a resource-constrained setting such as a government hospital. This study aimed to identify factors associated with mortality in patients with severe COVID-19 pneumonia admitted to a tertiary care COVID-19 hospital in South India.

Address for correspondence: Padukudru Anand Mahesh, Department of Respiratory Medicine, JSS Medical College, JSS University, Mysore, Karnataka, India; e-mail: mahesh1971in@gmail.com

DOI: 10.5603/ARM.a2021.0036 | Received: 06.01.2021 | Copyright (C 2021 PTChP | ISSN 2451-4934 | e-ISSN 2543-6031

This article is available in open access under Creative Common Attribution-Non-Commercial-No Derivatives 4.0 International (CC BY-NC-ND 4.0) license, allowing to download articles and share them with others as long as they credit the authors and the publisher, but without permission to change them in any way or use them commercially. 


\section{Material and methods}

\section{Study design}

We conducted a retrospective study at a tertiary care teaching hospital in South India. The study was approved by institutional ethics committee (SIMS/IEC/503/2020-21).

\section{Participants and eligibility criteria}

We retrospectively analyzed consecutive patients with severe COVID-19 pneumonia who had been admitted to our hospital from June to October 2020. Individuals aged 18 years and above were included in the study. Diagnosis of COVID-19 was defined as the patient having a positive result on the oropharyngeal and nasopharyngeal swab for SARS-CoV-2 by reverse transcriptase polymerase chain reaction (RTPCR). Our hospital used test kits provided by the government of Karnataka.

Classification of severity of SARS-CoV-2 infection was based on the revised national guidelines on clinical management of SARS-CoV-2 infection given by the Ministry of Health and Family Welfare, Government of India [4].

We defined severe COVID-19 pneumonia as an "adult with fever or suspected respiratory infection, plus one of the following; respiratory rate $>30$ breaths/min, severe respiratory distress, $\mathrm{SpO}_{2}<90 \%$ at room air".

\section{Data collection}

The records of patients admitted to high dependency unit and intensive care unit were collected and evaluated for predictors of mortality. Data included demographic details, symptoms and the duration of symptoms, comorbidities like diabetes, hypertension, heart disease, obesity, chronic kidney disease, chronic liver disease, malignancy, chronic respiratory diseases like asthma and Chronic Obstructive Pulmonary Disease. Clinical parameters like pulse rate, heart rate, blood pressure, peripheral capillary oxygen saturation/fraction of inspired oxygen $\left(\mathrm{SpO}_{2} / \mathrm{FiO}_{2}\right)$ on admission were collected. Laboratory parameters included complete hemogram (Sismex,6 part differential cell counter), neutrophil-lymphocyte ratio, random blood sugar, serum ferritin (Beckman), Serum Lactate Dehydrogenase (LDH) (ERBA EXEL 640), renal function test, liver function test, arterial blood gas, chest radiography. Treatment details like the use of remdesivir, the day of starting remdesivir after the symptom onset, the use of steroids, use of anticoagulants, use of high-flow nasal cannula, noninvasive ven- tilation, ventilator, incidence of hospital-acquired infections were collected. The outcome variables included the length of hospital stay and mortality.

The patients were managed with supportive care and specific pharmacological protocols created by the hospital's COVID-19 management guidelines committee in accordance with the government of Karnataka. Specific pharmacological treatments included systemic corticosteroids, low-molecular-weight heparin, oxygen and remdesivir. Data collected were cross-checked by the authors, and at the end of data entry - by an independent investigator. Any disagreement between two investigators was resolved by reaching a consensus.

A total of 4,012 patients with laboratory-confirmed SARS-CoV-2 were admitted during the study period. We excluded from the final analysis patients who were still receiving care in the hospital at the time of preparation of this manuscript and those with incomplete information.

\section{Statistical analysis}

Descriptive data are presented as frequencies (percentages) of discrete variables and as means (SDs) of continuous variables. For comparisons between the two groups, ANOVA test with the Bonferroni correction was used. $\chi^{2}$-test was applied to evaluate categorical factors. Fischer's exact test was used in case of low cell frequency. The Cox regression univariate and multinomial analysis and Kaplan-Meier analysis were used for survival investigation. The receiver operating characteristic (ROC) curves were constructed for age, duration of symptoms, $\mathrm{SpO}_{2} / \mathrm{FiO}_{2}$, serum ferritin, respiratory rate and the cut-off value with the highest sensitivity and specificity selected as threshold. All statistical tests were 2-tailed, and factors were considered statistically significant at $\mathrm{p}<0.05$. IBM SPSS version 22 and CDC Epi Info version 7 were used for analysis.

\section{Results}

A total of 4,012 confirmed cases of COVID-19 were admitted to hospital, of which $560(13.95 \%)$ with great severity were included in the study (Figure 1). Mean age of the study population was $57.75 \pm 13.96$ years. Three hundred sixty-five (65.17\%) were men. Hypertension (41.25\%) followed by diabetes (41\%) was the most common comorbidity. Dyspnea (69.46\%) was the most frequent symptom followed by fever (52.5\%) and cough (46.78\%) (Table 1). Mean duration of symptoms before admission 


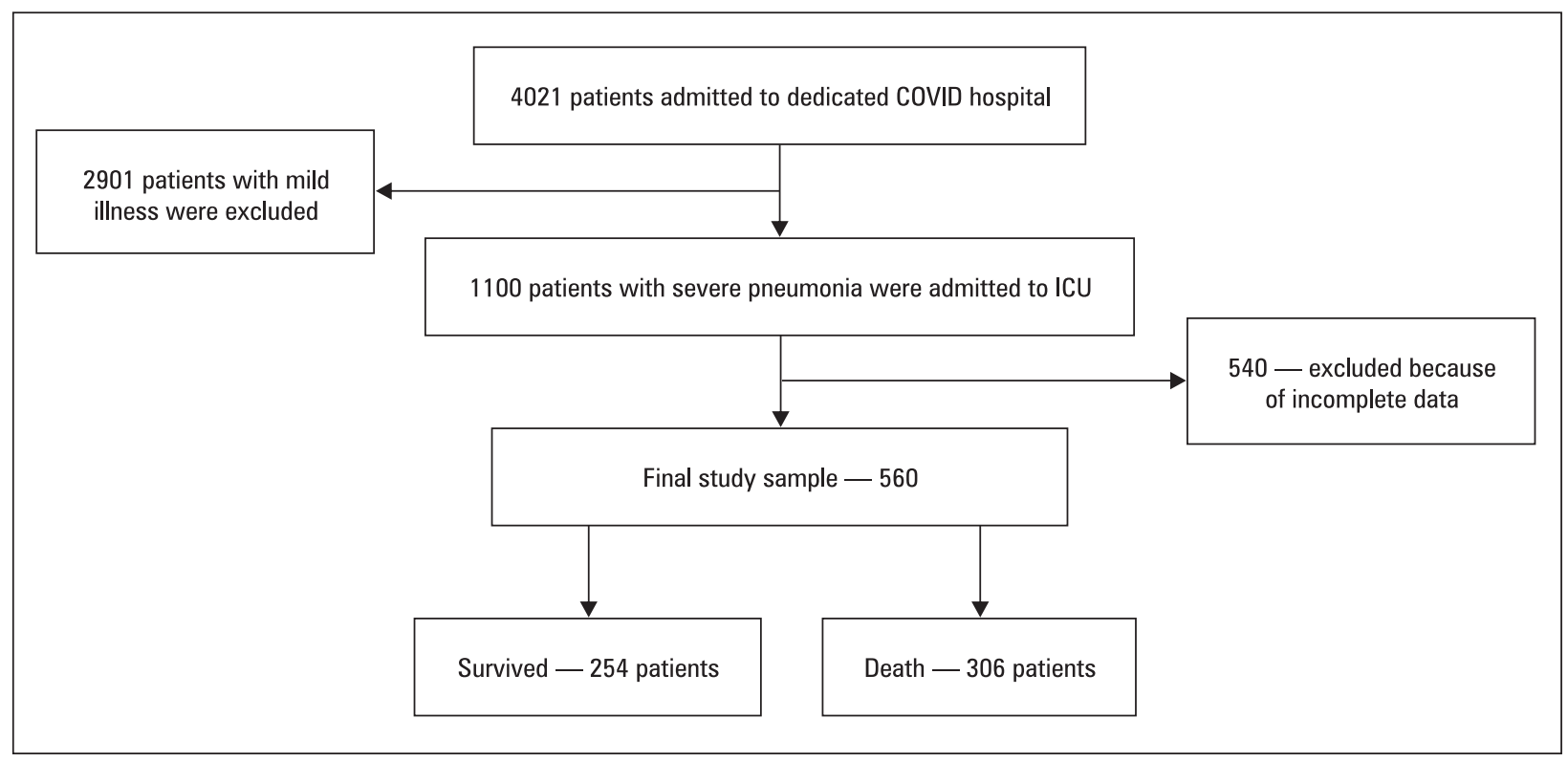

Figure 1. The flowchart depicting enrolment of COVID-19 patients into the study

was $4.11 \pm 2.09$ days. Remdesivir was given to 298 (53.21\%) patients. Mean duration of starting remdesivir after the symptom onset was $5.58 \pm$ 2.78 days. High-flow nasal cannula was given to 245 (43.7\%) subjects. Ninety-one (16.25\%) needed ventilatory support. A very high mortality, i.e. 306 (54.64\%) cases was observed at our hospital. Among the patients who died, nearly quarter of them (82 patients) died within 24-48 hours from admission. Secondary bacterial infection was noted at a late stage of the disease in 8 patients, and organisms isolated were Streptococcus pneumonia, Klebsiella pneumoniae and Staphylococcus aureus. In the Cox regression analysis, we observed age $>50$ years, duration of symptoms more than 4 days, $\mathrm{SpO}_{2} / \mathrm{FiO}_{2}<400$ on admission, serum ferritin $>450 \mu \mathrm{g} / \mathrm{L}$ on admission, respiratory rate $>23 / \mathrm{min}$ on admission, the presence of comorbidities and non-usage of remdesivir to be independent predictors of mortality in patients with severe COVID-19 pneumonia (Table 2). We observed an increased hazard of death by two days after the onset of symptoms which peaked on the $5^{\text {th }}$ day of the symptom onset. The risk of death then decreased, but remained significant till the $8^{\text {th }}$ day (Figure 2). We found steroid usage, use of mechanical ventilation and the day of starting remdesivir after the symptom onset to be independent predictors of prolonged hospitalization in patients with severe COVID-19 pneumonia (Table 3). The presence of chronic liver disease, use of mechanical ventilation, day of starting remdesivir after the symptom onset to be inde- pendent predictors of prolonged ICU stay in patients with severe COVID-19 pneumonia (Table 4). The Kaplan-Meier analysis showed statistically significant mortality benefit in patients who received remdesivir and even better survival if used within 4 days of the symptom onset (Figure 3A and $3 \mathrm{~B})$. Only a small number of subjects $(<2 \%)$ succumbed post-discharge due to COVID-19-related complications with a final mortality rate at 30 days of $56.60 \%$.

\section{Discussion}

In the present study, we found elderly patients with male predominance more commonly affected by moderate to severe pneumonia with very high mortality rates of more than $50 \%$. We observed age $>50$ years, duration of symptoms more than 4 days on admission, $\mathrm{SpO}_{2} / \mathrm{FiO}_{2}<400$ on admission, serum ferritin $>$ $450 \mathrm{mcg} / \mathrm{L}$ on admission, respiratory rate $>23 / \mathrm{min}$ on admission, the presence of comorbidities and non-usage of remdesivir to be independent predictors of mortality in patients with severe COVID-19 pneumonia.

Elderly patients are commonly affected by severe pneumonia due to age-dependent decline in immunity. A Korean meta-analysis of COVID-19 pneumonia found old age to be the risk factor for increased mortality [5]. Old age is an established risk factor for various infections, including viral infections and by far most significant predictor of mortality in COVID-19 pneumonia [6, 7]. 
Table 1. Baseline characteristics of patients with severe COVID-19 pneumonia admitted to ICU

\begin{tabular}{|c|c|c|c|c|}
\hline Variables & Total $(n=560)$ & Survived $(\mathrm{n}=254)$ & Death $(n=306)$ & P-value* \\
\hline Age [years], mean (SD) & $57.75(13.96)$ & $54.39(14.99)$ & $60.54(12.39)$ & 0.004 \\
\hline Gender, n [\%] & $365(65.17)$ & $166(65.35)$ & $199(65.03)$ & 0.840 \\
\hline \multicolumn{5}{|l|}{ Symptoms, n [\%] } \\
\hline Cough & $262(46.78)$ & $131(51.57)$ & $131(42.95)$ & 0.083 \\
\hline Dyspnea & $389(69.46)$ & $140(55.12)$ & 249 (81.37) & 0.001 \\
\hline Fever & $294(52.5)$ & $161(63.39)$ & $133(43.46)$ & 0.0001 \\
\hline Myalgia & $61(10.89)$ & $41(16.14)$ & $20(6.54)$ & 0.315 \\
\hline $\begin{array}{l}\text { Duration of symptoms before admission, } \\
\text { mean (SD) [in days] }\end{array}$ & $4.11(2.09)$ & $3.27(1.92)$ & $4.79(1.98)$ & 0.0001 \\
\hline Comorbidities, n [\%] & $343(61.25)$ & $115(45.28)$ & $228(74.51)$ & 0.0001 \\
\hline Diabetes, n [\%] & $230(41)$ & $80(31.50)$ & $150(49.02)$ & 0.016 \\
\hline Hypertension, n [\%] & $231(41.25)$ & $72(28.35)$ & $159(51.96)$ & 0.009 \\
\hline Ischemic heart disease, $\mathrm{n}[\%]$ & $48(8.5)$ & $11(4.33)$ & $37(12.09)$ & 0.118 \\
\hline Chronic kidney disease, $\mathrm{n}$ [\%] & $32(5.7)$ & $4(1.57)$ & $28(9.17)$ & 0.043 \\
\hline Chronic liver disease, $\mathrm{n}$ [\%] & $9(1.6)$ & $1(0.39)$ & $8(2.61)$ & 0.171 \\
\hline Morbid obesity, n [\%] & $13(2.3)$ & $1(0.39)$ & $12(3.92)$ & 0.092 \\
\hline \multicolumn{5}{|l|}{ Vitals } \\
\hline Sp02 at room air [on admission], mean (SD) & $78.70(18.72)$ & $87.74(12.17)$ & $71.19(19.87)$ & 0.0001 \\
\hline Respiratory rate, breath/min, mean (SD) & $21.37(4.82)$ & $19.94(2.97)$ & $22.5(5.67)$ & 0.003 \\
\hline \multicolumn{5}{|l|}{ Laboratory findings at the time of admission } \\
\hline Hemoglobin [gm\%], mean (SD) & $12.35(2.14)$ & $12.42(2.18)$ & $12.34(2.08)$ & 0.121 \\
\hline Total white blood cell count, mean (SD) & $9.87(6.5)$ & $9.04(4.59)$ & $10.56(7.74)$ & 0.071 \\
\hline Platelet count $\left[\mathrm{lakh} / \mathrm{mm}^{3}\right]$ & $2.10(0.93)$ & $2.24(0.87)$ & $2.03(0.86)$ & 0.081 \\
\hline Neutrophil Lymphocyte Ratio mean (SD) & $8.02(8.66)$ & $5.87(4.37)$ & $9.80(10.71)$ & 0.029 \\
\hline Serum ferritin $[\mu \mathrm{g} / \mathrm{L}]$, mean (SD) & $539.66(381.78)$ & $367.2(308.63)$ & $632.29(385.61)$ & 0.0001 \\
\hline $\begin{array}{l}\text { Serum Lactate dehydrogenase [LDH] [U/L], } \\
\text { mean (SD) }\end{array}$ & $845.73(593.51)$ & $788.1(681.62)$ & $866.39(558.52)$ & 0.160 \\
\hline Serum Creatinine, [mg/dl] mean (SD) & $1.66(2.08)$ & $1.24(1.33)$ & $1.94(2.51)$ & 0.002 \\
\hline Random blood sugar [mg/dl] mean (SD) & $215.49(135.3)$ & $181.32(112.48)$ & $239.92(144.8)$ & 0.002 \\
\hline \multicolumn{5}{|l|}{ Treatment } \\
\hline Remdesivir usage $n$ [\%] & $298(53.21)$ & $165(64.96)$ & $133(43.46)$ & 0.019 \\
\hline $\begin{array}{l}\text { First dose of Remdesivir after symptoms } \\
\text { onset, mean (SD) }\end{array}$ & $5.58(2.78)$ & $5.06(3.12)$ & $6.01(2.37)$ & 0.0001 \\
\hline Low-molecular-weight heparin, n [\%] & $365(65.17)$ & $154(60.63)$ & $211(68.95)$ & 0.161 \\
\hline Steroid usage $\mathrm{n}[\%]$ & $454(81)$ & $185(72.83)$ & $269(87.91)$ & 0.023 \\
\hline $\begin{array}{l}\text { First dose of steroid after admission [in } \\
\text { days], mean (SD) }\end{array}$ & $1.22(1.19)$ & $1.19(1.25)$ & $1.24(1.03)$ & 0.931 \\
\hline High-flow nasal cannula, n [\%] & $245(43.7)$ & $41(16.14)$ & $204(66.67)$ & 0.0001 \\
\hline Ventilator, n [\%] & $91(61.25)$ & $3(1.18)$ & $88(28.76)$ & 0.0001 \\
\hline No. of days in ICU, mean (SD) & $4.48(3.23)$ & $5.48(3.18)$ & $3.64(3.04)$ & 0.0001 \\
\hline No. of days of hospital stay, mean (SD) & $8.71(7.54)$ & $12.53(8.76)$ & $5.52(4.28)$ & 0.00001 \\
\hline
\end{tabular}

${ }^{*}$ ANOVA test with Bonferroni adjustment for multiple comparisons 
Table 2. Cox univariate and multivariate analysis of factors associated with mortality in patients with severe COVID-19 pneumonia

\begin{tabular}{|c|c|c|c|c|}
\hline Variables & $\begin{array}{l}\text { Hazard ratio } \\
(95 \% \mathrm{CI})\end{array}$ & P-value & $\begin{array}{c}\text { Adjusted hazard ratio } \\
\text { (95\% Cl) }\end{array}$ & P-value \\
\hline $\mathrm{SpO}_{2} / \mathrm{FiO}_{2}<400$ & $3.35(2.631-4.264)$ & 0.001 & $2.424(1.869-3.145)$ & 0.0001 \\
\hline Age $>50$ years & $1.01(1.00-1.02)$ & 0.0001 & $1.589(1.132-2.228)$ & 0.007 \\
\hline Duration of symptoms $>4$ days & $1.23(1.18-1.28)$ & 0.0001 & $2.410(1.659-3.502)$ & 0.0001 \\
\hline Serum ferritin $>450 \mu \mathrm{g} / \mathrm{L}$ & $1.001(1.001-1.001)$ & 0.0001 & $2.134(1.671-2.725)$ & 0.0001 \\
\hline Neutrophil to lymphocyte ratio $>7$ & $1.02(1.01-1.03)$ & 0.0001 & $1.122(0.880-1.429)$ & 0.354 \\
\hline First dose of remdesivir after symptom onset $>4$ days & $1.038(1.003-1.075)$ & 0.038 & $1.234(0.747-2.036)$ & 0.411 \\
\hline Respiratory rate $>23 / \mathrm{min}$ & $1.08(1.06-1.10)$ & 0.0001 & $1.343(1.046-1.725)$ & 0.021 \\
\hline Diabetes & $1.46(1.16-1.83)$ & 0.001 & $0.835(0.635-1.097)$ & 0.195 \\
\hline Hypertension & $1.12(1.02-1.23)$ & 0.014 & $0.874(0.754-1.012)$ & 0.072 \\
\hline Ischemic heart disease & $1.87(1.33-2.65)$ & 0.001 & $1.233(0.857-1.774)$ & 0.258 \\
\hline Chronic kidney disease & $2.35(1.59-3.48)$ & 0.0001 & $1.383(0.886-2.158)$ & 0.153 \\
\hline Presence of any comorbidity & $2.32(1.79-3.0)$ & 0.0001 & $1.822(1.286-2.581)$ & 0.001 \\
\hline Remdesivir usage & $0.75(0.59-0.93)$ & 0.013 & $0.453(0.342-0.599)$ & 0.0001 \\
\hline Steroid usage & $1.73(1.22-2.44)$ & 0.001 & $1.097(0.749-1.608)$ & 0.633 \\
\hline Creatinine $>1.5 \mathrm{mg} / \mathrm{dl}$ & $1.08(1.04-1.12)$ & 0.001 & $1.161(0.884-1.524)$ & 0.284 \\
\hline
\end{tabular}

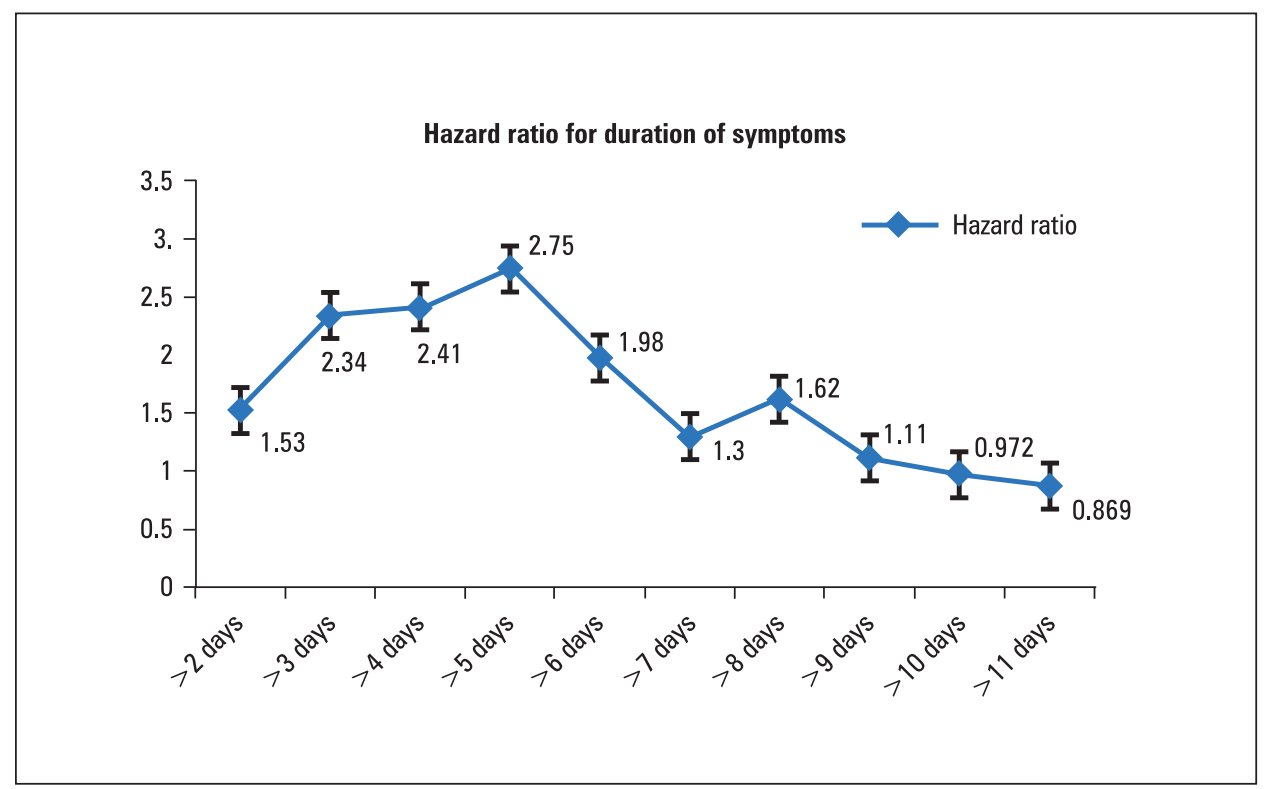

Figure 2. The graph depicting hazard ratio of mortality for each day from the symptom onset and duration of hospitalization

Elderly patients infected with SARS-CoV-2 tend to trigger hyper-activation of the immune system and hypercoagulation in small blood vessels leading to cytokine storm [8]. Though it is still unclear why the elderly are more prone to cytokine storm, possible mechanisms include an increase in activity and abundance of NLRP-3 (Nucleotide-binding oligomerization domain, Leucine rich Repeat and Pyrin domain containing protein 3), a component of inflammasome in immune cells and alveolar macrophages in the lungs which upon chronic stimulation cause pulmonary fibrosis [9]. NLRP-3 activity is normally under control of Sirtuin-2 (protein implicated in longevity) which reduces with age. This decline in Sirtuin-2 is exacerbated by SARS-CoV-2 infection and might 
Table 3. Cox univariate and multivariate analysis of factors associated with prolonged hospital stay more than $\mathbf{5}$ days in patients with severe COVID-19 pneumonia

\begin{tabular}{lcccc}
\hline Variables & $\begin{array}{c}\text { Hazard ratio } \\
(\mathbf{9 5 \%} \mathbf{~ C l})\end{array}$ & $\begin{array}{c}\text { P-value } \\
\text { Fever }\end{array}$ & $\begin{array}{c}\text { Adjusted hazard ratio } \\
\mathbf{( 9 5 \% ~ C l )}\end{array}$ & P-value \\
Dyspnea & $0.72(0.581-0.90)$ & $\mathbf{0 . 0 0 4}$ & $0.804(0.638-1.014)$ & 0.066 \\
Comorbidities & $1.26(1.01-1.58)$ & $\mathbf{0 . 0 3 6}$ & $1.04(0.806-1.356)$ & 0.738 \\
First dose of Remdesivir after symptom onset $>4$ days & $1.28(1.03-1.59)$ & $\mathbf{0 . 0 2 5}$ & $1.17(0.930-1.17)$ & 0.177 \\
Steroid usage & $0.959(0.929-0.989)$ & $\mathbf{0 . 0 0 8}$ & $0.932(0.899-0.966)$ & $\mathbf{0 . 0 0 0 1}$ \\
Use of ventilator & $1.29(0.98-1.71)$ & $\mathbf{0 . 0 6}$ & $1.42(1.030-1.976)$ & $\mathbf{0 . 0 3 2}$ \\
\hline
\end{tabular}

Table 4. Cox univariate and multivariate analysis of factors associated with prolonged ICU stay more than 4 days in patients with severe COVID-19 pneumonia

\begin{tabular}{|c|c|c|c|c|}
\hline Variables & Hazard ratio $(95 \% \mathrm{CI})$ & P-value & $\begin{array}{l}\text { Adjusted hazard ratio } \\
\text { (95\% Cl) }\end{array}$ & P-value \\
\hline Fever & $0.670(0.520-0.864)$ & 0.002 & $0.77(0.588-1.008)$ & 0.062 \\
\hline Dyspnea & $1.38(1.07-1.80)$ & 0.014 & $1.18(0.884-1.598)$ & 0.254 \\
\hline Ischemic heart disease & $1.59(0.998-2.55)$ & 0.05 & $1.45(0.869-2.44)$ & 0.154 \\
\hline Chronic kidney disease & $1.91(0.981-3.73)$ & 0.05 & $1.50(0.751-3.019)$ & 0.249 \\
\hline Chronic liver disease & $9.49(2.97-30.35)$ & 0.0001 & $6.58(1.94-22.27)$ & 0.002 \\
\hline Comorbidities & $1.44(1.12-1.86)$ & 0.004 & $1.20(0.904-1.614)$ & 0.202 \\
\hline First dose of Remdesivir after symptom onset $>4$ days & $0.956(0.923-0.989)$ & 0.015 & $0.940(0.904-0.977)$ & 0.002 \\
\hline Use of ventilator & $3.35(2.15-5.23)$ & 0.0001 & $3.23(2.031-5.152)$ & 0.0001 \\
\hline
\end{tabular}

promote hyperactivation of NLRP3 and trigger cytokine storm in elderly patients [10]. Another possible reason for increased susceptibility to COVID-19 infection in the elderly could be due to a decrease in T-cells and subsets, which reduces with aging [11]. Mahase et al. found that overall death rate from COVID-19 was $0.66 \%$ and was sharply rising to $7.8 \%$ in elderly people aged over 80 years [12].

Ferritin is an intracellular protein that stores iron and releases in a controlled fashion. Apart from the role of iron store, it has a potential capacity during inflammation following SARSCoV-2 infection. Ferritin is found to be secreted by alveolar macrophages in the lungs and also stimulated by various cytokines, including IL-6 [13]. Active ferritin in turn stimulates the immune system and activates macrophages leading to an increase in inflammatory process [14]. Various single-center retrospective studies done in China found higher ferritin levels in patients who succumbed compared to survivors and discovered a decrease in ferritin levels with remission of the disease [15-17]. We also found elevated ferritin levels in non-survivors (632.29 $\mu \mathrm{g} / \mathrm{L})$ compared to survivors $(367.2 \mu \mathrm{g} / \mathrm{L})$.

We noted $\mathrm{SpO}_{2} / \mathrm{FiO}_{2}(\mathrm{SF})$ ratio on admission $<400$ to be an independent predictor of mortality in severe COVID-19 pneumonia patients. $\mathrm{SpO}_{2} / \mathrm{FiO}_{2}$ has been used as a surrogate prognostic marker of $\mathrm{PaO}_{2} / \mathrm{FiO}_{2}$ in acute respiratory distress syndrome (ARDS) patients with similar characteristics and the outcome in the previous study [18]. According to the Kigali modification, ARDS was defined without the need for positive end-expiratory pressure (PEEP), with the presence of bilateral opacities in the chest radiograph and hypoxia defined with a cut-off of $\mathrm{SpO}_{2} / \mathrm{FiO}_{2}$ less than or equal to 315 [19]. The study done by Riviello et al. using Kigali modification of the Berlin definition had good correlation with the diagnosis of ARDS [20]. $\mathrm{SpO}_{2} / \mathrm{FiO}_{2}$ is one of noninvasive parameters that might predict a poor outcome in patients with severe SARS-CoV-2 infection [21]. $\mathrm{SpO}_{2} / \mathrm{FiO}_{2}$ ratio could be used for correct estimation of ARDS in developing countries like India, 
A

Survival Functions

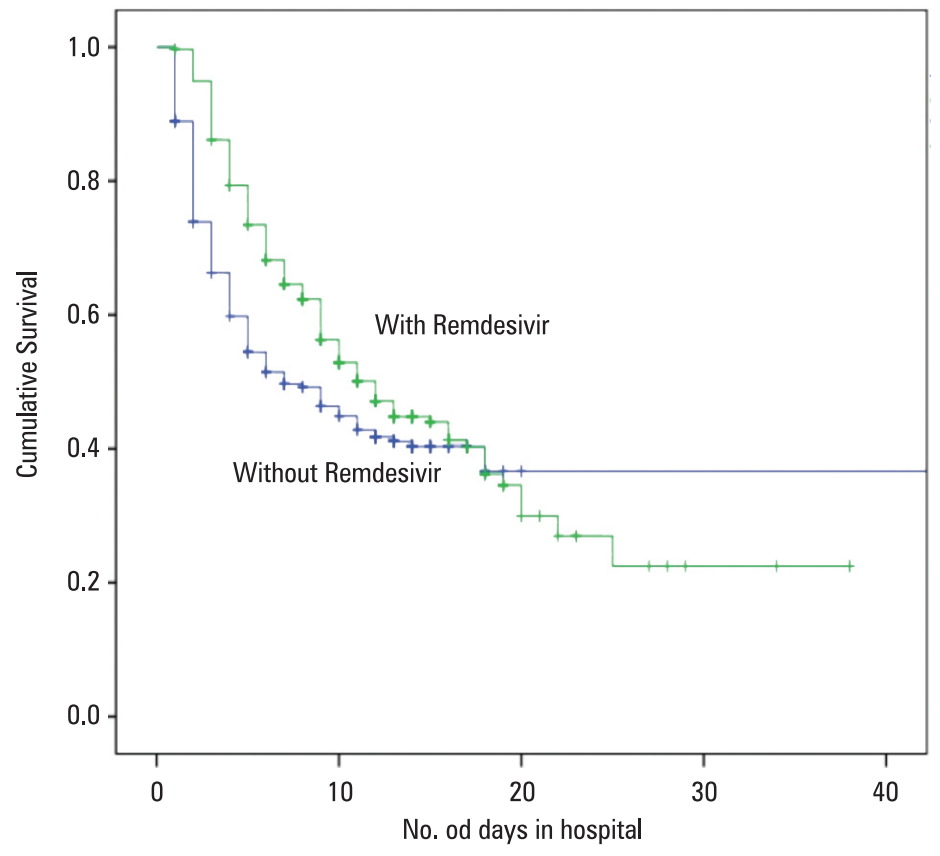

Remdesivir Usage

$\neg 0 \quad \neg 1 \quad \longrightarrow$ Without Remdesivir $\longrightarrow$ With Remdesivir

B

Survival Functions

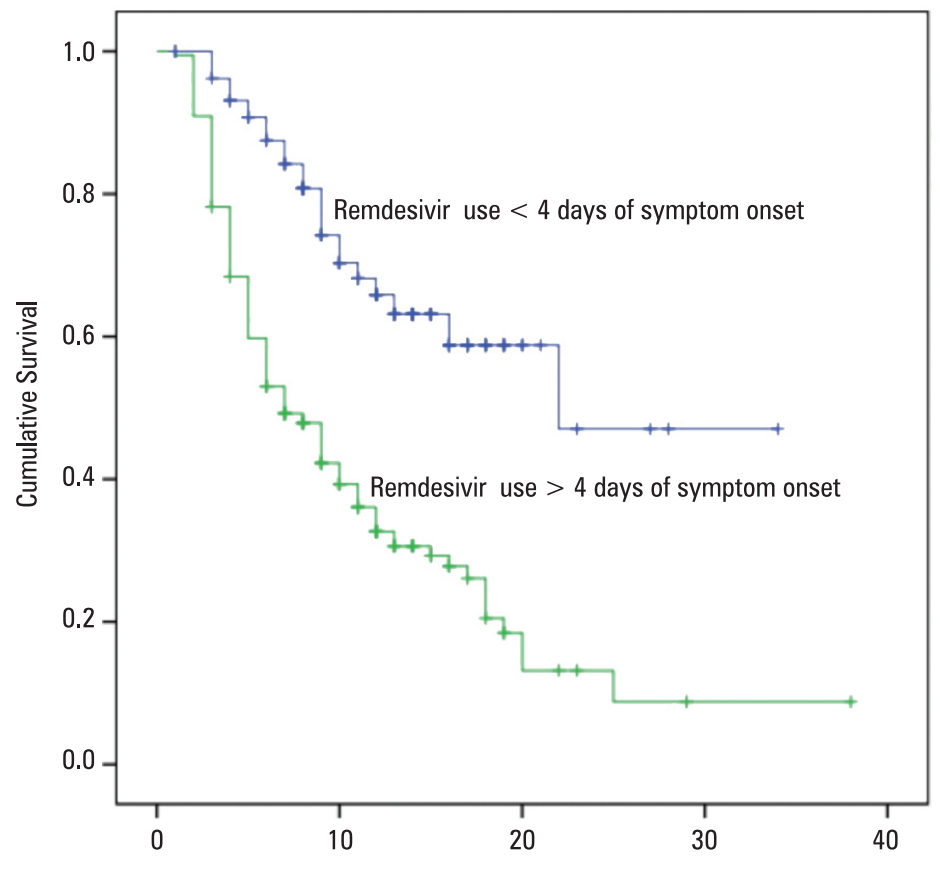

No. od days in hospital

Symptom onset $>4$ days

$\neg 0 \longrightarrow 1 \longrightarrow 0-<4$ days of symptoms $\longrightarrow 1-<4$ days of symptoms

Figure 3. A. The Kaplan-Meier graph for survival with the use of remdesivir in patients with severe COVID-19 pneumonia; B. The Kaplan-Meier graph for survival with remdesivir use before and after 4 days of the symptom onset in patients with severe COVID-19 pneumonia 
where there is scarcity of critical care specialist and intensive care in the periphery, especially in the COVID-19 pandemic situation.

Several antiviral drugs have been evaluated for the treatment of SARS-CoV-2 infection, but no antiviral agents have shown any mortality benefit. Remdesivir, a nucleoside analog with broad antiviral activity among RNA viruses, including Ebola, has been tried for treatment of SARS-CoV-2 infection. It acts by interfering with non-structural protein 12 polymerase (nsp12) which is a multisubunit of RNA synthesis complex that is responsible for viral RNA genome replication. Remdesivir has shown to decrease time to recovery in adults hospitalized with lower respiratory tract infection in an preliminary study of randomized control trial [22], and may prevent progression to more severe disease. The final report showed that remdesivir improved mortality rates for those receiving supplemental oxygen ( $4 \%$ with remdesivir versus $13 \%$ with placebo on day 29 of treatment) [23]. A Chinese study by Wang et al. showed numerical reduction in time to improvement with remdesivir compared to placebo, however, it was not statistically significant [24]. A randomized controlled trial (RCT) done by Spinner et al. also observed early clinical improvement in patients on remdesivir compared to standard care [25]. None of the RCTs has shown mortality benefit from usage of remdesivir.

The median duration of starting remdesivir in our study was 5.50 days compared to studies done by Spinner et al., Beigel et al., Wang et al which was 8 days, 9 days and 11 days, respectively [22, $24,25]$. In the above mentioned clinical trials, the benefit of remdesivir was larger when given earlier in the illness. Like other viral infections (eg.influenza), early use of antiviral drug is associated with improved clinical outcome [26]. Whether the use of remdesivir early in the course of disease when viral replication is the most active and complications have not yet occurred, would improve outcomes - remains to be confirmed by larger RCT studies.

The presence of comorbidities is an established risk factor for mortality in patients with COVID-19 pneumonia in various studies done across the globe [27-30]. We observed comorbidities in nearly $61 \%$ of patients. Hypertension $(41.25 \%)$ and diabetes $(41 \%)$ were the most commonly noted. Nearly $66.5 \%$ of patients with comorbidities succumbed to illness. A recent meta-analysis done in India found the prevalence of hypertension (22.9\%) the highest among COVID-19 patients, and diabetes was more prev- alent in the Indian population compared to other countries [30]. One of the largest Chinese studies ( $\mathrm{n}=72,314)$ found significantly increased mortality in COVID-19 patients with comorbidity [31]. The latest report from the Center for Disease Control, United States discovered cardiovascular diseases (including hypertension, stroke, coronary artery disease, cardiac failure) in $60.9 \%$ of patients with COVID-19 [32]. A UK study observed cardiac disease, chronic obstructive pulmonary disease, chronic kidney disease, obesity and liver disorders to be associated with a significant increase in mortality [33]. A recent report by the Ministry of Health and Family Welfare, India that analyzed the death of 15,962 patients with SARS-CoV-2 infection found the presence of one or more comorbidities in $57 \%$ of patients [34]. Unlike other studies, our Cox multinominal analysis did not provide evidence for an association between specific comorbidity and mortality.

Vital parameters play an important role in initial assessment and triaging of patients with pneumonia. Respiratory rate being one of the components of many severity scoring systems like CURB65 (Confusion, Urea, Respiratory rate, Blood pressure, Age $>65$ years) score, APACHE II (Acute Physiology And Chronic Health Evaluation-II) score shows its importance. We found increased respiratory rate $(>23 / \mathrm{min})$ to be an independent risk factor for mortality similar to a large American study [35].) A Chinese study on 344 critically ill patients also found higher respiratory rate was associated with poor outcome indicating more attention to be paid to vital signs [21].

Due to COVID-19 pandemic, there are accelerated publications without long-term follow-up of patients with mortality data [36].) There is sparse information on mortality rates post-discharge in severe COVID-19 pneumonia in the Indian population. We observed mortality rate of $56.60 \%(n=560)$ in patients with severe COVID-19 pneumonia at 30 days which is lower than that from studies done in Pakistan (n-204, 77\%) [37], the United States (n-373, 75.6\%) [38] and China (n-344, 88.3\%) [21]. However, studies carried out by Graselli et al. in Italy (n-1,581, 26\%) [39]) and Gupta et al. in the United States (n-2,215, 35.4\%) [40] had lower ICU mortality than our study. A possible explanation for the disparity in mortality rates is that around $58 \%$ and $28 \%$ of the study cohort was still in ICU without an outcome projecting falsely low mortality in the studies done by Graselli and Gupta, respectively. In a study conducted by Zhou et al. (n-50) who 
followed up all patients till the outcome, the mortality rate was $78 \%$ [41].

\section{Strengths and limitations}

One of the important strengths of our study is the presence of 30 days mortality data post-discharge which is lacking in many studies of patients with severe COVID-19 pneumonia. The evaluation of the timing of remdesivir administration after the symptom onset on mortality rates is an important finding as many studies which did not show any benefit of remdesivir had administered the drug late in the course of the disease. Limitations of our study include the usual limitations of a retrospective study, furthermore, information was collected from a single center.

\section{Conclusion}

We found age $>50$ years, the duration of symptoms more than 4 days, $\mathrm{SpO}_{2} / \mathrm{FiO}_{2}<400$ on admission, serum ferritin $>450 \mu \mathrm{g} / \mathrm{L}$ on admission, respiratory rate $>23 / \mathrm{min}$ on admission, the presence of comorbidities and non-usage of remdesivir and late initiation of remdesivir after the symptom onset to be independent predictors of mortality in patients with severe COVID-19 pneumonia.

\section{Clinical implication/future directions}

Mortality predictors found in the study could be identified early and treated to possibly reduce mortality in severe COVID-19 pneumonia patients. Mortality benefits of remdesivir with early initiation in the course of the disease need to be relooked with large randomized controlled trials.

\section{Conflict of interest}

None declared.

\section{References:}

1. Timeline: WHO's COVID-19 response [Internet]. Available from: https://www.who.int/emergencies/diseases/novel-coronavirus-2019/interactive-timeline. [Last accessed: November 20, 2020].

2. CSSEGISandData / COVID-19. Available online: https://github. com/CSSEGISandData/COVID-19. [Last accessed: November 19, 2020].

3. Wikipedia. COVID-19 pandemic data. Available online: https:// en.wikipedia.org/w/index.php?title=Template:COVID-19_pandemic_data\&oldid $=989520654$. [Last accessed at: November 19, 20리.

4. Revised COVID-19 treatment for COVID-19 in India. Google Search. Available online: www.google.com/search?q=REVISED + COVID-19 + TREATMENT + FOR + COVID-19 + IN + INDIA\&oq = REVISED + COVID-19+ + TREATMENT +FOR +COVID-19+IN+INDIA\&aqs $=$ chrome..69i57j33i22i29i3 $0.23070 j 0 j 7 \&$ sourceid $=$ chrome\&ie $=U$ UTF-8. [Last accessed at:
November 19, 2020].

5. Kim W, Han J, Lee K. Predictors of mortality in patients with COVID-19: a systematic review and meta-analysis. Korean Journal of Clinical Pharmacy. 2020; 30(3): 169-176, doi: 10.24304/kjcp.2020.30.3.169.

6. Williamson E, Walker A, Bhaskaran K, et al. OpenSAFELY: factors associated with COVID-19-related hospital death in the linked electronic health records of 17 million adult NHS patients. , doi: 10.1101/2020.05.06.20092999.

7. Santesmasses D, Castro J, Zenin A, et al. COVID-19 is an emergent disease of aging. Aging Cell. 2020; 19(10), doi: 10.1111/ acel.13230.

8. Terpos E, Ntanasis-Stathopoulos I, Elalamy I, et al. Hematological findings and complications of COVID-19. Am J Hematol. 2020; 95(7): 834-847, doi: 10.1002/ajh.25829, indexed in Pubmed: $\underline{32282949}$.

9. Stout-Delgado HW, Cho SJ, Chu SG, et al. Age-Dependent susceptibility to pulmonary fibrosis is associated with NLRP3 inflammasome activation. Am J Respir Cell Mol Biol. 2016; 55(2): 252-263, doi: 10.1165/rcmb.2015-02220C, indexed in Pubmed: 26933834.

10. Kouhpayeh Sh, Shariati L, Boshtam M, et al. The Molecular Story of COVID-19; NAD+ Depletion Addresses All Questions in this Infection. Preprint available online: https:// www.preprints.org/manuscript/202003.0346/v1. 2020, doi: 10.4324/9780203528709-10.

11. Diao Bo, Wang C, Tan Y, et al. Reduction and functional exhaustion of t cells in patients with coronavirus disease 2019 (COVID-19). Front Immunol. 2020; 11: 827, doi: 10.3389/fimmu.2020.00827, indexed in Pubmed: 32425950.

12. Mahase E. Covid-19: death rate is $0.66 \%$ and increases with age, study estimates. BMJ. 2020; 369: m1327, doi: 10.1136/bmj. m1327, indexed in Pubmed: 32238354.

13. Rosário C, Zandman-Goddard G, Meyron-Holtz EG, et al. The hyperferritinemic syndrome: macrophage activation syndrome, Still's disease, septic shock and catastrophic antiphospholipid syndrome. BMC Med. 2013; 11: 185, doi: 10.1186/1741-7015-11-185, indexed in Pubmed: 23968282.

14. Hyperferritinemia and inflammation - PubMed [Internet]. [Last accessed at: November 15, 2020]. Available from: https:// pubmed ncbi nlm nih gov. ; 28541437.

15. Chen G, Wu Di, Guo W, et al. Clinical and immunological features of severe and moderate coronavirus disease 2019. J Clin Invest. 2020; 130(5): 2620-2629, doi: 10.1172/JCI137244, indexed in Pubmed: $\underline{32217835}$.

16. Ji D, Zhang D, Chen Z, et al. Clinical characteristics predicting progression of COVID-19. SSRN Electronic Journal. , doi: 10.2139/ssrn.3539674.

17. Zhou F. Clinical course and risk factors for mortality of adult in patients with COVID-19 in wuhan, china: a retrospective cohort study. Lancet. 2020; 395(10229): 1054-1062, doi: 10.1016/ S0140-6736(20)30566-3, indexed in Pubmed: $\underline{32171076}$.

18. Chen W, Janz D, Shaver C, et al. Clinical characteristics and outcomes are similar in ARDS diagnosed by oxygen saturation/f io 2 ratio compared with pao 2 /F io 2 ratio. Chest. 2015; 148(6): 1477-1483, doi: 10.1378/chest.15-0169.

19. Lazzeri C, Peris A. The Kigali modification of the berlin definition: a new epidemiological tool for ARDS? J Thorac Dis. 2016; 8(6): E443-E445, doi: 10.21037/jtd.2016.03.84, indexed in Pubmed: 27294247.

20. Riviello ED, Kiviri W, Twagirumugabe T, et al. Hospital incidence and outcomes of the acute respiratory distress syndrome using the kigali modification of the Berlin definition. Am J Respir Crit Care Med. 2016; 193(1): 52-59, doi: 10.1164/rccm.201503-05840C, indexed in Pubmed: 26352116.

21. Wang Y, Lu X, Li Y, et al. Clinical course and outcomes of 344 intensive care patients with COVID-19. Am J Respir Crit Care Med. 2020; 201(11): 1430-1434, doi: 10.1164/rccm.202003-0736LE, indexed in Pubmed: $\underline{32267160}$.

22. Beigel JH, Tomashek KM, Dodd LE, et al. Remdesivir for the treatment of COVID-19 - final report. N Engl J Med. 2020; 383(19): 1813-1826, doi: 10.1056/NEJMoa2007764, indexed in Pubmed: $\underline{32445440}$.

23. Final report confirms remdesivir benefits for COVID-19. National Institutes of Health (NIH). Available from: https://www. 
nih.gov/news-events/nih-research-matters/final-report-confirms-remdesivir-benefits-covid-19. [Last accessed: November 26, 2020].

24. Wang Y, Zhang D, Du G, et al. Remdesivir in adults with severe COVID-19: a randomised, double-blind, placebo-controlled, multicentre trial. The Lancet. 2020 May 16. ; 395(10236): 1569-78.

25. Spinner CD, Gottlieb RL, Criner GJ, et al. Effect of remdesivir vs standard care on clinical status at 11 days in patients with moderate COVID-19: a randomized clinical trial. JAMA. 2020; 324(11): 1048-1057, doi: 10.1001/jama.2020.16349, indexed in Pubmed: $\underline{32821939}$.

26. Timing of Remdesivir for COVID-19 | The Medical Letter, Inc. Available from: https://secure.medicalletter.org/w1609a. [Last accessed at: November 26, 2020].

27. EpiCentro. Characteristics of COVID-19 patients dying in Italy. Available from: https://www.epicentro.iss.it/en/coronavirus/ sars-cov-2-analysis-of-deaths. [Last accessed at: November 26, 2020].

28. CDC COVID-19 Response Team. Preliminary estimates of the prevalence of selected underlying health conditions among patients with coronavirus disease 2019 - United States, February 12-March 28, 2020. MMWR Morb Mortal Wkly Rep. 2020; 69(13): 382-386, doi: 10.15585/mmwr.mm6913e2, indexed in Pubmed: 32240123 .

29. Chen T, Wu Di, Chen H, et al. Clinical characteristics of 113 deceased patients with coronavirus disease 2019: retrospective study. BMJ. 2020; 368: m1091, doi: 10.1136/bmj.m1091, indexed in Pubmed: 32217556.

30. Singh AK, Misra A. Impact of COVID-19 and comorbidities on health and economics: Focus on developing countries and India. Diabetes Metab Syndr. 2020; 14(6): 1625-1630, doi: 10.1016/j.dsx.2020.08.032, indexed in Pubmed: 32891011.

31. Wu Z, McGoogan JM. Characteristics of and Important Lessons From the Coronavirus Disease 2019 (COVID-19) Outbreak in China: Summary of a Report of 72314 Cases From the Chinese Center for Disease Control and Prevention. JAMA. 2020; 323(13): 1239-1242, doi: 10.1001/jama.2020.2648, indexed in Pubmed: $\underline{32091533}$.

32. Wortham J, Lee J, Althomsons S, et al. Characteristics of persons who died with COVID-19 - United States, February 12-May 18, 2020. The COVID-19 Reader. 2020: 152-164, doi: 10.4324/9781003141402-17.
33. Docherty AB, Harrison EM, Green CA, et al. Features of 20133 UK patients in hospital with COVID-19 using the ISARIC WHO Clinical Characterisation Protocol: prospective observational cohort study. BMJ. 2020; 369: m1985, doi: 10.1136/bmj.m1985, indexed in Pubmed: 32444460.

34. $43 \%$ of Covid patients in India who died had no comorbidities: Govt analysis/. Available from:https://theprint.in/health/43-ofcovid-patients-in-india-who-died-had-no-comorbidities-govtanalysis/453687/. [Last accessed at: Noveber 27, 2020].

35. Bahl A, Van Baalen MN, Ortiz L, et al. Early predictors of in-hospital mortality in patients with COVID-19 in a large American cohort. Intern Emerg Med. 2020; 15(8): 14851499, doi: 10.1007/s11739-020-02509-7, indexed in Pubmed: 32970246 .

36. Chaddha U, Kaul V, Agrawal A. What is the true mortality in the critically ill patients with COVID-19? Indian J Crit Care Med. 2020; 24(6): 383-384, doi: 10.5005/ip-journals-10071-23435, indexed in Pubmed: 32863627.

37. Rahim F, Amin S, Noor M, et al. Mortality of patients with severe COVID-19 in the intensive care unit: an observational study from a major COVID-19 receiving hospital. Cureus. 2020; 12(10): e10906, doi: 10.7759/cureus.10906, indexed in Pubmed: 33194473.

38. Richardson S, Hirsch JS, Narasimhan M, et al. Presenting characteristics, comorbidities, and outcomes among 5700 patients hospitalized with COVID-19 in the New York City Area. JAMA 2020; 323(20): 2052-2059, doi: 10.1001/jama.2020.6775, indexed in Pubmed: 32320003.

39. Grasselli G, Zangrillo A, Zanella A, et al. Baseline characteristics and outcomes of 1591 patients infected with SARS-CoV-2 admitted to icus of the Lombardy Region, Italy. JAMA. 2020; 323(16): 1574-1581, doi: 10.1001/jama.2020.5394, indexed in Pubmed: $\underline{32250385}$.

40. Gupta S, Hayek SS, Wang W, et al. Factors associated with death in critically ill patients with coronavirus disease 2019 in the US. JAMA Intern Med. 2020 [Epub ahead of print], doi: 10.1001/jamainternmed.2020.3596, indexed in Pubmed: $\underline{32667668 .}$

41. Zhou F, Yu T, Du R, et al. Clinical course and risk factors for mortality of adult inpatients with COVID-19 in Wuhan, China: a retrospective cohort study. The Lancet. 2020; 395(10229): 1054-1062, doi: 10.1016/s0140-6736(20)30566-3. 